\title{
Comparison of Photodetector Performances in a Color Filtered Spectrophotometer
}

\author{
V.G. BOCEKCI* AND H. KORKMAZ \\ Marmara University, Department of Electrical and Electronics Engineering, Technology Faculty, \\ 34722 İstanbul, Turkey
}

In this study, the performances of phototransistor and phototube that can be used as a photodetector in a spectrophotometer device are compared. The spectrophotometer used is at a basic level and filters out the desired wavelength from broadband spectrum light through the color filter. With color filter, 16 different wavelength colors can be obtained. The filtered light falls onto the photodetector after passing through the pure water solution. It has been determined that the performance of the phototransistor is much higher in the experiments in which the phototransistor and phototube are used separately. The results of the experiments show that both the output amplitude and the measurement resolution are higher.

DOI: 10.12693/APhysPolA.134.329

PACS/topics: optoelectronics, spectrophotometer, photodetector

\section{Introduction}

A spectrophotometer is the main measurement equipment in areas like physics, biotechnology and food engineering where measurements are required on samples [1]. A component called photodetector is used in spectrophotometers to measure the light intensity from the light source in order to determine whether the substance absorbs the light. Photodetectors have wide spectral responses from ultraviolet to visible and from visible to near infrared. Thanks to these characteristics, it has a wide range of applications from industrial to scientific areas [2].

Selection of the appropriate detector is critical for measuring the light intensity accurately and reliably. The detector should be suitable with the spectra of the beams that need to be detected. The selected detector should be sensitive to the minimum values of the beams included in the system [3]. Desired characteristics of a photodetector are its sensitivity to the light, its capability to generate a signal directly proportional with the light intensity, its fast response to a light that falls on it, i.e. its ability to generate the highest output signal level as possible in a shorter duration by using auxiliary circuits.

The study conducted by Kim et al. suggests a system to measure color, wavelength, and purity by using a simple LED and photodiode instead of the standard spectrophotometry elements. The system they designed is based on a color measurement principle and capable of measuring even smaller samples [1].

Widdess et al., on the other hand, compared in their study the spectrophotometers that use a phototube, photodiode, and charge-coupled device (CCD) as the actively

*corresponding author; e-mail: vgbocekci@marmara.edu.tr utilized sensing element in the market. The spectrophotometer that uses photodiode gives more competitive results compared to a phototube and has a larger measurement wavelength range. It is also more favorable in terms of cost [4].

In spectrophotometric systems, another element that can be used as a detector is phototransistor. Phototransistor is a kind of optic transducer that senses and amplifies the amount of light. Another advantage of this transducer is its ability to work under low voltage conditions with better noise performances [5].

The purpose of this study is to compare the performances of phototube that is used frequently in spectrophotometer devices and the phototransistor element which is seen more frequently in new generation of devices.

\section{Materials and method}

In this study, a modular spectrophotometer system was used to evaluate the performances of two types of photodetectors. The evaluations were performed by analyzing the corresponding voltages of light passing through the pure water solution at different wavelengths.

\subsection{Basic mechanism of the spectrophotometer}

3Ds MAX model of the simple spectrophotometer device used during the measurements is shown in Fig. 1. The spectrophotometer consists of a tungsten halogen lamp featuring a broad band as an illuminant, a filter layer containing 16 color filters to obtain lights at various wavelengths, an experimental solution stage and finally a photodetector stage for converting the light into voltage.

\subsection{Photodetector layer}

In the spectrophotometer developed, two different types of sensing elements were used respectively, a phototransistor as shown in Fig. 2a and a phototube as shown 


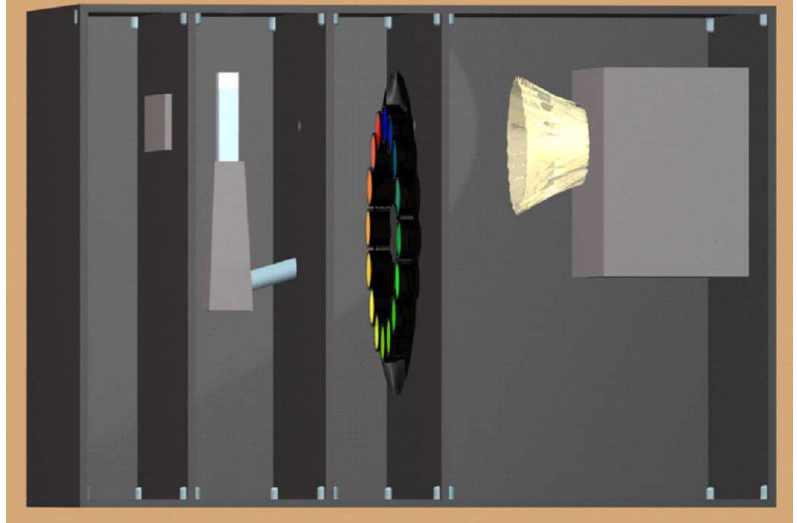

Fig. 1. 3Ds MAX model showing basic layers of the designed spectrophotometer device.
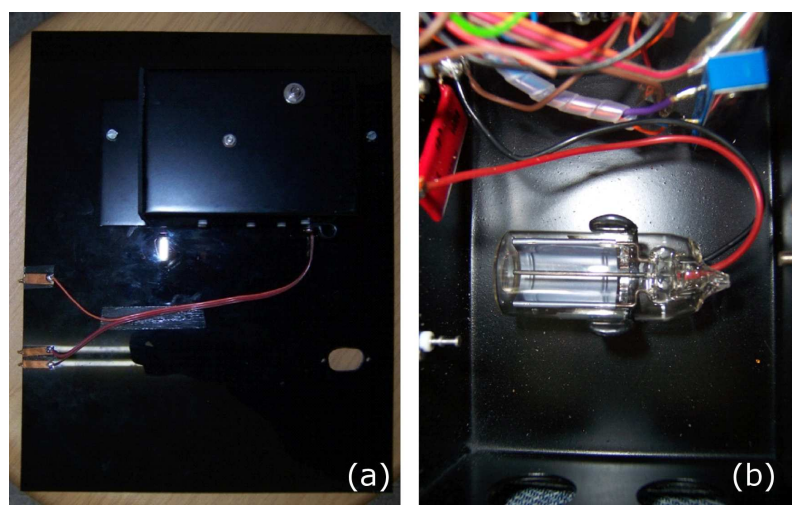

Fig. 2. The setup showing the detector types used in the spectrophotometer device: (a) phototransistor, (b) phototube.

in Fig. 2b. The light received by the photodetector layer is converted into electrical signal by a linear lightmeter circuit and then amplified. This circuit converts the incident light on itself into a voltage level. Output voltage of the circuit decreases as the light intensity over the phototransistor used in the circuit falls. Schematic diagram of the linear light meter circuit can be seen in Fig. 3 .

\section{Results}

In the experiments conducted, broad spectrum light was first applied through a filter selected among 16 different color filters at 400-700 $\mathrm{nm}$ wavelengths, and pure water was chosen as the reference sample. When the light falls onto the photosensitive element, it converts the signal into electrical form. In our study, output voltages of linear light meter circuit were measured in separate experiments conducted under the same circumstances to be able to compare the performances of photosensitive elements. Measurement results can be seen in Table I.

The voltage values acquired from both of two photo sensitive elements were compared as illustrated in Fig. 4.

Measurements with phototransistor have a peak value at $580 \mathrm{~nm}$. Phototube signal variation, on the other hand, is less than the phototransistor and stays almost

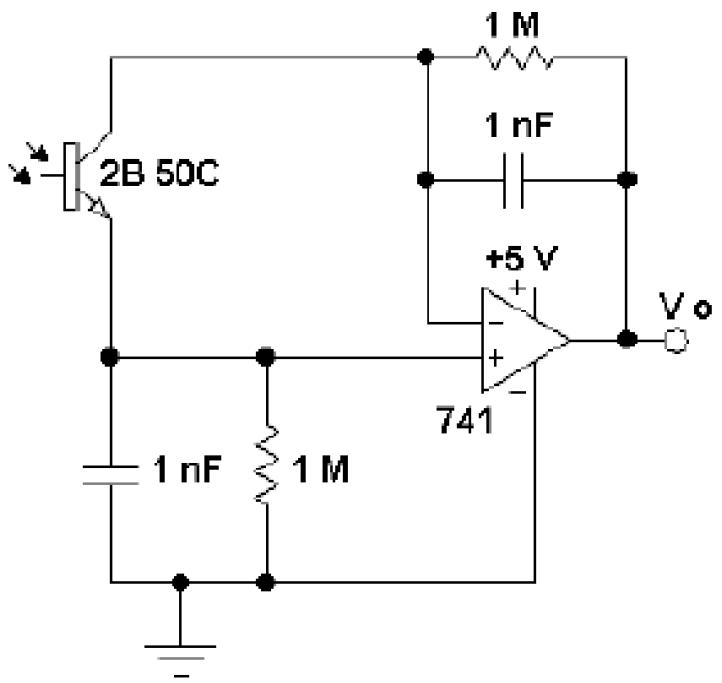

Fig. 3. Schematic diagram of the linearlight meter circuit.

\section{TABLE I}

Output voltage of transistor and phototube detectors using pure water

\begin{tabular}{c|c|c}
\hline $\begin{array}{c}\text { Wavelength } \\
\lambda[\mathrm{nm}]\end{array}$ & $\begin{array}{c}\text { Voltage measured } \\
\text { by phototransistor }[\mathrm{V}]\end{array}$ & $\begin{array}{c}\text { Voltage measured } \\
\text { by phototube }[\mathrm{V}]\end{array}$ \\
\hline 400 & 1.961 & 1.634 \\
420 & 1.965 & 1.678 \\
440 & 2.071 & 1.645 \\
460 & 2.173 & 1.704 \\
480 & 2.172 & 1.539 \\
500 & 2.243 & 1.706 \\
520 & 2.497 & 1.491 \\
540 & 2.461 & 1.513 \\
560 & 2.475 & 1.724 \\
580 & 3.249 & 1.502 \\
600 & 2.461 & 1.678 \\
620 & 2.536 & 1.704 \\
640 & 2.705 & 1.594 \\
660 & 2.883 & 1.536 \\
680 & 2.901 & 1.528 \\
700 & 2.405 & 1.481
\end{tabular}

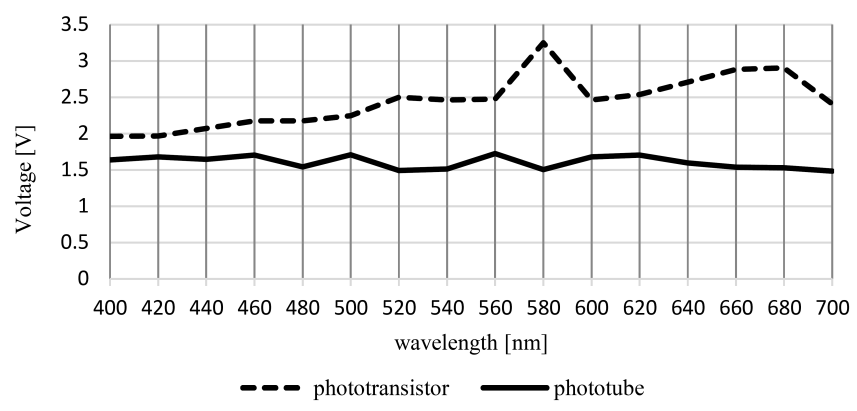

Fig. 4. Comparison of two different types of photosensitive elements. 
constant along a particular interval. Furthermore, the measurement results shows that resolution of the phototube was lower than the phototransistor.

\section{Conclusion}

One of the most significant stages used in spectrophotometer devices to measure the substance concentration in liquid samples is the photodetector and its signal conditioning circuit. In our study, two different types of photodetector performances were compared by using acquired signal from both of phototube and phototransistor elements under the same circumstances. Obtained results indicate that the resolution of measurements acquired from the phototube were lower than the phototransistor.

\section{References}

[1] J.S. Kim, A.H. Kim, H.B. Oh, B.J. Goh, E.S. Lee, J.S. Kim, J.H. Jun, Bio-Med. Mater. Eng. 26, S1773 (2015).

[2] J. Yoo, S. Jeong, S. Kim, J.H. Je, Adv. Mater. 27, $1712(2015)$.

[3] E. Musayev, Optoelektronik Devreler ve Sistemler, Birsen Pub., Istanbul 1999 (in Turkish).

[4] K. Widdess, D. Herrick, S. Wolf, D. Goggin, B. Pedra Neme, W. Barros, D. Carter, in: Research \& Creative Endeavor Symp., 2015.

[5] I.H. Chen, S.S. Tseng, P.W. Li, IEEE Photon. Technol. Lett. 21, 1674 (2009). 